\title{
Impacts of Military- Endurance- Solid- Sport- Beverage in the Function on the Physical Training
}

\author{
Jianfu Zhu ${ }^{1,}$, Qiaozhen Yan ${ }^{1}$ \\ ${ }^{1}$ School of Physical Education, Wenshan University, Wenshan 663099, China \\ paperiset@163.com
}

Keywords: Military- Endurance- Solid- Sport-Beverage, Physical Training, Impact Indicators

\begin{abstract}
With the booming training, military- endurance- solid- sport-beverage gets more and more people's attention. Physical effects of various nutrients for athletes recover quickly reviewed, the method of training during sports supplement food nutrition and dietary antioxidant in sports and physical fatigue effects, rapid recovery of different projects personalized nutritional needs. Militaryendurance- solid- sport-beverage without blood and cellular immune significant impact, but can improve the function of the level of heavy load training of officers and men, the effect carbohydrate supplement drinks and no insulin response. Reflects the amount of training and load index of blood urea and serum change kinase training next morning, the experimental group increased magnitude lower than the control group increased amplitude, indicating the depth of the beverage can effectively hang athletic fatigue, can significantly enhance training effectiveness.
\end{abstract}

\section{Introduction}

The body proper nutrition, good nutrition structure, mainly refers to the proportion of essential nutrients appropriate, and the necessary material contains sufficient nutrients to the principles guiding the deployment of the diet, which is called a balanced diet. Specifically, a balanced diet means that while athletes make in four areas between dietary nutrient supply and establish a balance between the body's physiological requirements, namely amino acid balance, heat balance of nutrients constitution, and a variety of acid-base balance between nutrient intake balance, only that help absorb and use nutrients for optimum ratio of the chemical composition of the human body to maintain. During exercise, the body needs more energy intake and oxygen consumption increased, which led to an increase in free radicals will be doubled, to reach up to a thousand times. The body had to consume large amounts of antioxidant vitamin E to repair the extra free radicals. Therefore, food and nutrition experts suggest: athletes after high-intensity exercise is best to take the right amount of vitamin E supplements and foods rich in vitamin $\mathrm{E}$, wheat germ, wheat germ products.

\section{Materials and methods}

\section{Nutrition}

Body functions and physical energy and reduce the load by a temporary decline in the state after the load back and exceeded the level before the process is called military- endurance- solid- sportbeverage process. Athletes military- endurance- solid- sport-beverage mainly relies adjust the amount of exercise, physical therapy and massage. Proper nutrition is an important factor in winning athlete, is to ensure the health of athletes and athletic ability, and nutrition for athletes training, function status, physical adaptation, recovery and movement disorders are closely related [Pitkanen, 2003]. Fig.1 shows logical structure of the nutrition system. 


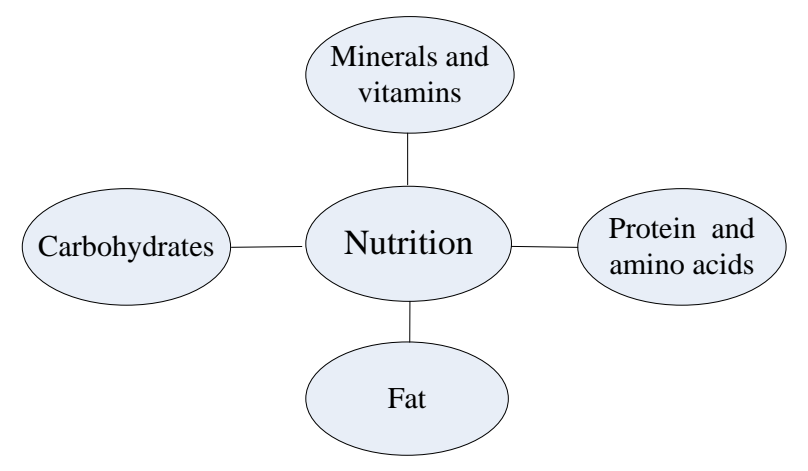

Figure 1. The basic model of nutrition

Carbohydrates (sugars short) are a major player in the energy supply of food substances for. Human tissue sugar content of about $2 \%$ of body weight, but the energy the body needs about $60 \%$, the latest research on military- endurance- solid- sport-beverage demonstrated by the supply of sugar in the diet, carbohydrates have the best results for the athletes to play an important role, high-intensity endurance training athletes carbohydrate intake accounted for $60 \%$ of the heat energy than a $70 \%$. 60 to 90 minutes after making a big load of training, almost run out of glycogen reserves, under normal mixed diet, can only be to make up for about 72 hours later. However, if the supplemental carbohydrate-rich foods, then the glycogen reserves in the 24 hours after the end of the load that is able to restore the original level. This case shows that good nutrition for physical recovery process is very important [Tsali, 2004].

Fat is high energy materials, heat production per unit weight of fat for carbohydrate and protein than twice. Late prolonged endurance exercise mainly depends on fat for energy, but do not digest fat, metabolic oxygen consumption is high, so the proportion of fat in the diet is too high will affect the supply of oxygen and fat metabolism more acidic substances, athletes endurance and recovery after physical activity detrimental. Generally believed that fat for energy a $30 \%$ to $25 \%$ of the total heat supply, the daily diet containing 50 grams of fat generally meet human needs, endurance athletes may be increased, but must be noted that the diet of fish, meat, etc. different types, including the amount of fat is different, different conditioning methods used grease should also be different, otherwise easily lead to excessive fat intake.

When the athlete's body has enough sugar and fat, protein is not involved in supplied-energy, and its role is to update the body proteins. Dietary amino acids are not stored in the body, most of them will quickly degrade, which require a sufficient number and because they contain a variety of amino acids in protein to replenish. Protein is an important component of the body's tissues constitute different exercise intensity and movement, human demand for protein is also different. In the case of a large amount of exercise, not only consume a lot of energy, but also makes the body protein breakdown strengthen even negative oxygen balance. Therefore, in order to ensure the quality and quantity of the protein intake of the supplement athletes' loss, increase muscle strength, and promote the synthesis of hemoglobin and accelerate recovery from fatigue will have great significance.

Athletes at high temperature or high humidity or large amount of exercise lasting endurance sports, mainly rely on a lot of sweat to achieve the effect of body heat. The loss of a large amount of sodium chloride with sweat during exercise, if not add will appear pathological phenomena such as muscle spasms, loss of calcium ions more easily lead to inadequate intake such as muscle cramps, fatigue, decreased bone density and fractures. Vitamins are the maintenance of human life and can regulate metabolism,; may lack nutrients, vitamin deficiency, the body live power weakened, lowered immunity, resulting in reduced exercise capacity [Dubnovq, 2004].

\section{Food Supplements}

Military- endurance- solid- sport-beverage are a class of nutrient composition and content can be adapted to the athletes or sports exercise physiological characteristics of the population, with special nutritional needs, regulate the body balance and soft drinks can improve exercise capacity, it can 
provide enough water and energy for the body quickly. With the development of sports, sugar electrolyte drinks has been widely used, containing 20\% Effect of drinking the right amount of sugar and salts drinks oligosaccharides in human experiments showed that drinks can maintain blood glucose oligosaccharides at higher levels, and stabilize blood volume, serum insulin, serum magnesium, potassium and blood lactate, increased exercise acting and time.

Premotor and supplementary motor military- endurance- solid- sport-beverage, for muscle, brain and other organs to provide energy for the body to maintain fluid balance and prevent dehydration provide water, this need not only for the long endurance exercise plays an important role, but also for in short-term high-intensity intermittent exercises. Cyclists make sugar found immediately after exercise carbohydrate supplement can increase blood glucose levels after 30min, and promote the recovery of blood lactate. When high-intensity exercise, increasing the demand for carbohydrates, but if excessive intake of carbohydrates can lead to weight gain, the same effect on improving athletic performance. Coyle and other detailed study of the amount of carbohydrate in endurance sports, and concluded that eating $30 \sim 60 \mathrm{~g}$ carbohydrate per hour, which can effectively support the ability to endurance exercise [Ward KD, 2004].

Fluid in the body plays a synthesis and decomposition energy substances transported nutrients, oxygen, and take action on behalf of the radio product to regulate body temperature, is extremely important in maintaining physiological functions. If not timely infusion will cause a lot of sweat after dehydration and electrolyte imbalance, not only the athlete's physical decline, but also affect the normal life of metabolism, hazardous to their health. In the case of a lot of sweat should use hypotonic or isotonic drinks, the temperature $5{ }^{\circ} \mathrm{C}$ to $15{ }^{\circ} \mathrm{C}$ an appropriate, timely rehydration after the game, the way it should be a small number, in order to speed up physical recovery.

Macromolecular polysaccharide solid military endurance military- endurance- solid- sport-beverage using biological preparation, the advantage of low osmolality value, low resistance components, digestion and absorption rate can be as high as 97\%, good stomach emptying effect, no insulin response characteristics. Electrolytes can be maintained due to the large amount of exercise training, a lot of sweat electrolyte deficiency caused maintain electrolyte balance in the body, and the solid electrolyte of potassium and sodium beverage calcium and vitamin in maintaining the body's normal physiological and motor function plays an important and irreplaceable role. Taurine can promote the secretion of pituitary hormones, activation of pancreatic function, thereby improving the state of the body's endocrine system, the body with beneficial metabolic regulation; and promote organism immunity enhancement and anti-fatigue. Taurine can be combined with the insulin receptor and promote cellular uptake and utilization of glucose, accelerated glycolysis.

Taurine prevention of iron deficiency anemia have a significant effect, it can not only promote the intestinal absorption of iron, but also increase the stability of the erythrocyte membrane; Taurine also has antioxidant, anti-aging effects, and thus can inhibit caused by elevated serum enzymes. This could also be the main reason for the experimental group kinase lower than the control group. Taurine as essential amino acids, with a clear anti-lipid peroxidation is a -protective agent. The military- endurance- solid- sport-beverage contain ingredients, physical training police officers to improve blood sugar steady and significant role in improving performance, but police officers to improve the immune cells and serum enzymes eliminate obvious aspects of the role, which requires future research and development in the multi-beverage attention and research strengthened. [Fudge, 2006].

\section{The Effect of Military- Endurance- Solid- Sport-Beverage}

Athletes during exercise in stress, the body produces a large amount of free radicals and cause damage to the cell membrane lipid peroxidation, is one of the important mechanisms of fatigue occurred. Therefore, as soon as possible to eliminate free radicals athlete fatigue is one of the key factors in the recovery. In recent years, a number of vitamins and mineral salts as a non-enzymatic antioxidants become a hot research, they are VE, VC, carotene, selenium, zinc, and taurine.

$\mathrm{VE}$ is recognized as fat-soluble vitamins have antioxidant effects, it is through a direct effect on a variety of oxygen free radicals, such as singlet oxygen, superoxide radicals and lipid peroxides, interrupt the chain reaction of lipid peroxidation, protection biofilms from lipid peroxides damage. 
Therefore, the body's complement VE can reduce oxygen free radical damage. The amount of added VE day is necessary, during anaerobic endurance training, recruitment VE day should be 100 to 400 international units. VC has a variety of antioxidant activity, involved in the body's lightweight, can scavenge superoxide anion radicals, free radicals and reactive derivatives light active groups.

The immune system in maintaining a stable internal environment, to fend off the invasion of pathogens has an extremely important role. Body through non-specific immune response and to identify antigen-specific immune response and antigen-expressing cells produce effects. All of CD3 $+\mathrm{T}$ cells express antigens. T cells can be divided into CD4 +, CD8 + cell population and so on. According to its function, can be divided into T helper cells (TH, CD4 +), T killer cells (TC, CDB +) and T suppressor cells ( $\mathrm{T} \mathrm{s}, \mathrm{CDB}+$ ) and other major subsets. According CD3 +, CD4 +, CD8 + positive rate of cells to determine people total T cells, T auxiliary / inducer cells (TH)) percentage subsets and CD4 + / CD8 ratios and T killer / suppressor cells / + cells in order to determine the immune status.

Sugar is the main source of energy during exercise muscle substance. Sources have blood sugar in the body, muscle glycogen and liver glycogen. Muscle glycogen and glycogen in the body is very limited, and blood sugar is more limited. Therefore, in the long endurance exercise of endogenous glycogen may soon be exhausted, especially skeletal muscle glycogen depletion, and lower blood glucose concentrations, can cause fatigue. Thus, exogenous glucose supplement becomes extremely important. Studies have shown that exogenous sugar supplement may comprise about $10-30 \%$ of the total carbohydrate oxidation during exercise, can provide energy accounting for about 16-20\% of the total energy consumption. This study confirmed that the solid military endurance sports drinks, glycemic effect, maintain good blood glucose results. Solid military endurance sports drinks not only can effectively complement the persistent lack of energy in the body, and can maintain a high blood sugar level, and then converted to a steady stream of glycogen and muscle glycogen, the muscles can withstand long hours of work to delay the occurrence of fatigue.

\section{Analysis of Impact Indicators}

Reasons for different sports and different training methods fatigue are different, so the militaryendurance- solid- sport-beverages are also differences. Red blood cell (RBC) is the main physical components of blood, and hemoglobin $(\mathrm{H} \mathrm{b})$ is the core material of oxygen transport links. HI, content reflect people's aerobic capacity, a significant impact on the athletic ability, Hb content of the influencing factors of nutrition, exercise stress, rest and so suffer. Large amount of Hb exercise training early athletes decline. From the blood indicators, the control group and the experimental group showed a significant change in the same, indicating that complement solid military endurance sports drinks, to enhance the role for $\mathrm{HI}$ ) significant enough. Therefore, food and nutrition experts suggest: athletes after high-intensity exercise is best to take the right amount of vitamin $\mathrm{E}$ supplements and foods rich in vitamin E, wheat germ, wheat germ products. Wrestling requires greater strength and coordination of the nervous system, and requires a short time to produce explosive force, making this type of movement severe hypoxia, and oxygen debt is greater than the nitrogen metabolism strong [Watson, 2005]. Fig.2 shows wrestlers sports.

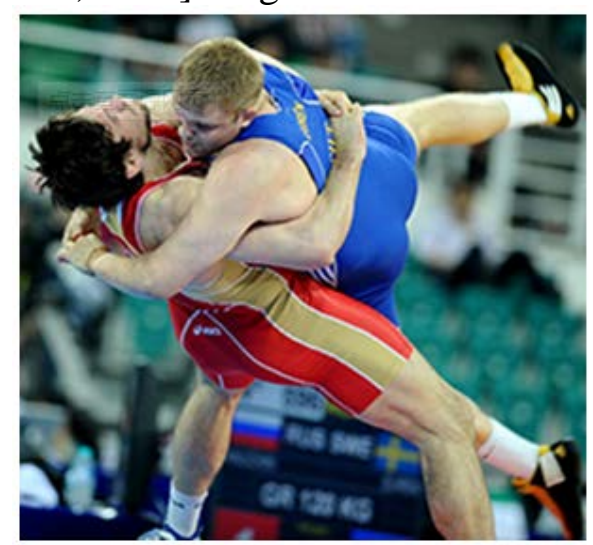

Figure 2. The wrestler sports 
In the large amount of exercise training, the body's blood biochemistry will change accordingly, which is the body's adaptive process for sports training stress. Alanine aminotransferase (ALT) mainly exists in a variety of tissues and cells, liver cells, the most abundant. Normally, only a very small amount released into the bloodstream, so the serum enzyme activity is low. Large amount of exercise or high intensity training is one of the causes appear high ALT day phenomenon. Serum aspartate aminotransferase (AST) in normal circumstances, the cells present in the tissue, wherein the myocardial cells of the highest concentrations, followed by the liver. Lactate dehydrogenase $(\mathrm{LDH})$ is in the process of energy metabolism is an important enzyme, present in all cardiac tissue, skeletal muscle and the like. LDH will rise when the skeletal muscle injury. Marathon and basketball sports athletes significantly increase serum LDH after exercise.

Basketball skills classes compete against the project belongs to a longer exercise duration, time is short and frequent intermittent movement, exercise intensity and volume are large, their energy supply is characterized by both aerobic anaerobic, energy consumption and lactic acid substances accumulate in the muscles and the blood is the main basketball player fatigue generated. Therefore, nutritional supplements after the basketball game, in addition to water and minerals, should immediately take 100-150g glucose. After the game two or three days should maintain a high calorie diet, rich in easily absorbed sugars and protein, lower fat content, multi vitamin supplements[Heaney, 2011]. Fig.3 shows basketball sports.

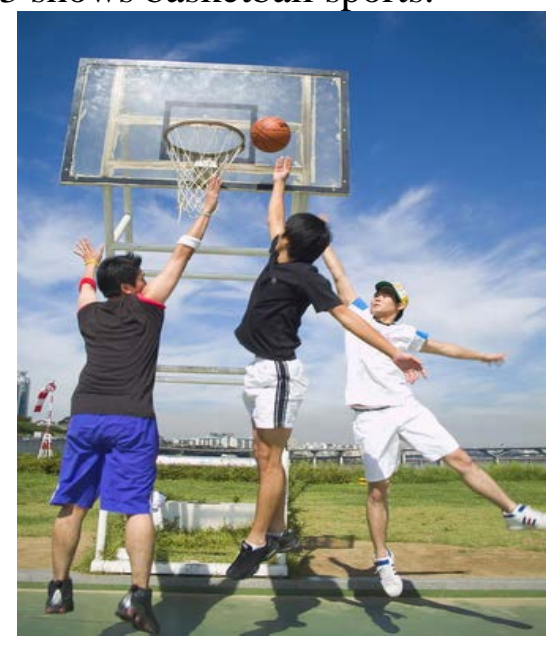

Figure 3 . The basketball sports

Long-distance running is a typical cyclical rate, speed and strength, speed, stamina and technique of combining physical class project. Technical characteristics of long-distance running is active front leg swing and the ground, do not stress after kicking knee straight, with emphasis on kicking swing, step length and stride frequency suitable fast. When the lack of (inadequate intake of dietary sugars or higher protein intake) of sugar, protein and amino acid catabolism strengthened, such as illness or exercise stress stimuli will lead to increased production of urea in the blood leaving the elevated levels. Kinase involved in the control of glycolysis, mitochondrial respiration and muscle contraction energy supply is one of the key enzymes in the body's energy supply system ATP- CP metabolism. Due to a mechanical exercise training so that environmental changes within the muscle cells, muscle cell membrane permeability increases muscle cells CIA then released into the circulating blood, serum CIA will cause different degrees of activity increased. CIA activity in serum after training, the recovery may reflect initial training load size and the body to adapt to the situation. When the heavy load training 2-3 days, serum CIA is still higher than $300 \mathrm{U} / \mathrm{L}$, showed a larger load, the body has not yet fully recovered. The next morning after exercise, serum CIA police officers are higher than 300U/L, and the control group was significantly higher than the experimental group, indicating greater strength training exercise load, and the next morning was not fully recovered, but the experimental group CIA recovery was significantly better than the control group [Burke, 2013]. Fig.4 shows long-distance running sports. 


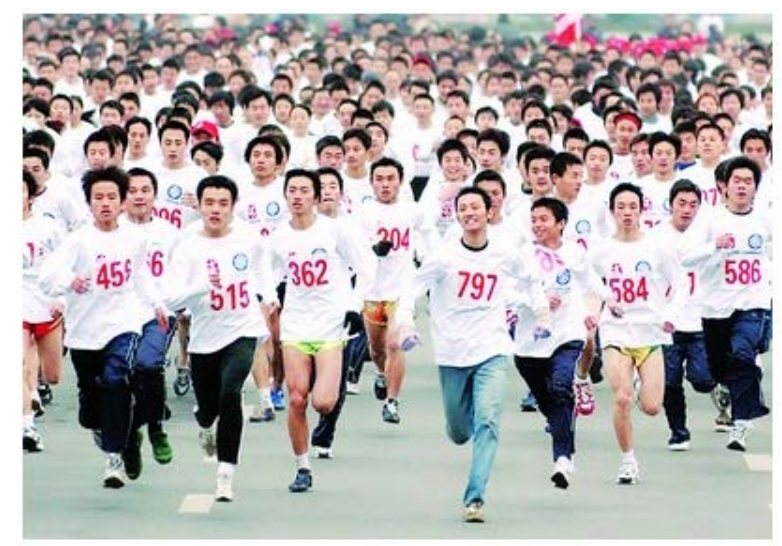

Figure 4. The long-distance running sports

\section{Conclusion}

The human body is a complex organism, while maintaining the life and activities require the certain energy consumption, exercise training can't do without the supply of energy, and the movement of energy sources of nutrients. Military- endurance- solid- sport-beverage can increase nutrition knowledge, implementation of scientific catering, strengthen the study of special nutritional problems, regularly check the nutritional status, the study of nutrition and training, rehabilitation and training the relationship between the various aspects of a comprehensive conduct, will achieve the desired results. Appropriate to add sugar, fat, protein and amino acids as well as vitamins and other minerals and nutrients for the rapid restoration of physical athlete has an important role, and in the training and exercise also need to add some nutrients. Due to movement of the body in stress, and therefore need to add certain antioxidants.

\section{References}

[1] Pitkanen HT, Oja SS, Rusko H, Numrnela A, Komi PV, Saransaari P, Takala T, Mero AA, 2003. Leucine supplementation does not enhance acute strength or running performance, but affects serum amino acid concentration. Amino Acids. 25(1), 85-94.

[2] Tsali s q Nikolaidis Mq Mougios V, 2004. Effects of iron intake through food or supplement on iron status and performance of healthy adolescent swimmers during a training season. Int $\mathrm{J}$ Sports Med. 25(4) ,306-13.

[3] DubnovqConstantini NW, 2004, Prevalence of iron depletion and anemia in top-level basketball players, Int J Sport NutrExerc Metab, 14(1), 30-37.

[4] Ward KD, Hunt KM, Berg MB, Slawson DA, Vukadinovich CM, McClanahan BS, 2004. Clemens LH, Reliability and validity of a brief questionnaire to assess calciumintake in female collegiate athletes, Int J Sport Nutr Exerc Metab, 14(2), 209-221.

[5] Fudge BW, Westerterp KR, Kiplamai FK, 2006. Evidence of negative balance using doubly labelled water in elite Kenyan endurance runners prior to competition. Br J Nutr, 95(1), 59-66.

[6] Watson TA, Blake RJ, Canister R, Garg ML, 2005. Antioxidant-restricted diet reduces plasma nonesterified fatty acids in trained athletes. Lipids,40(4), 433-435.

[7] Quatrowoni P. A, 2008. Clinical observations frc;m nutrition services in college athletics. J Am Diet Assoc, 108(4): G 89-694.

[8] Heaney, S, H. O'Connor, 2011,ca al. Nutrition knowledge in athletea: a systematic review. Int J sport Nutr Exerc Mctab, 21(31: 248-261. 
[9] Doyle-Lucas A.F and B. M. Davy, 2011. Development and evaluation of an educational intervention program for pre-professional adolesc;:nt ballet dancers: nutrition for optimal performance. J Dance Med Sci 5(2): 65-75.

[10] Valliant M .W H. P, 2012. Emplaincourt et al.Nutrition education by a registered dietitian improves dietary intake and nutrition knowledge of a NCAA female, ollevball team. $\mathrm{N}$ utrients, 4(6):506-516

[11]Burke, L. M N. L. Meyer, et al, 2013,. National nutritional programs for the 2012 London Olympic games: a systematic approach by three different countries. Nestle Nutr Inst Workshop Ser, 76: 103-120.

[12]Burkhart, S. J. and P. C. Pelly, 2013, Athlete use and opinion of point of choice nutrition labels at a major international competition. Appetite 70: 6- 13.

[13] Xie Wei, Ma Jiachen, Yang Mingli, 2010, “Design of hybrid architecture for intelligent service mobile robot", Proceeding of international cocnference on electrical and control engineering, pp 740-743. 